\title{
Mirando atrás para seguir avanzando. Una reflexión crítica sobre el pasado y el presente de la atención en salud mental (II)
}

Looking back to keep moving forward. A critical reflection on the past and present of mental health care (II)

\section{Marcelino López Álvarez}

Psiquiatra y sociólogo.

Director del comité de expertos de FAISEM.

Correspondencia: marcelino.lopezal@gmail.com

Recibido: 16/01/2019; aceptado con modificaciones: 18/11/2019

Resumen: Este es el segundo de una serie de dos artículos que tratan de hacer una valoración crítica de los principales aspectos teóricos y técnicos relacionados con la atención en salud mental que se han desarrollado en las 6 o 7 últimas décadas, como alternativa a dos tipos de posiciones consideradas inadecuadas, la del reduccionismo biomédico imperante y la de algunas tendencias nuevamente "antipsiquiátricas". En este segundo artículo se valoran algunos movimientos interesantes de estos últimos años y se plantean algunas conclusiones y perspectivas de futuro, tratando de situarse en una perspectiva crítica razonable desde el marco de la atención comunitaria en salud mental. Considerada esta como el paradigma tecnológico desde el que cabe dar cuenta de la complejidad de los problemas de salud mental y ayudar a resolverlos en una interacción no menos compleja entre profesionales y usuarios y usuarias.

Palabras clave: atención comunitaria en salud mental, rehabilitación psicosocial, antipsiquiatría, movimientos de usuarios y usuarias. 


\begin{abstract}
This is the second of two papers that seek to make a critical assessment of the main theoretical and technical aspects related to mental health care developed in the last six or seven decades, as an alternative to two types of positions considered inadequate; namely, that of the dominant biomedical reductionism and those of some new "antipsychiatric" tendencies. In this second paper some interesting movements from recent years are valued and some conclusions and prospects for the future are raised, trying to take a reasonable critical stance from the community mental health care framework. This latter one considered as the technological paradigm from which it is possible to take into account the complexity of mental health problems and to solve them in a no less complex interaction between professionals and users.
\end{abstract}

Key words: community mental health care, psychosocial rehabilitation, antipsychiatry, users' movements.

\title{
UNA VALORACIÓN CRÍTICA DE ALGUNOS MOVIMIENTOS DE INTERÉS
}

$\mathrm{L}$ a VAloración del desarrollo teórico y técnico de la atención en salud artículo anterior (1), debe tener en cuenta también la aparición de distintos movimientos, profesionales y no profesionales, que han modificado en diverso sentido y trascendencia el panorama. Todos surgieron en el marco de países europeos y de América del Norte, extendiéndose progresivamente a otras regiones, incluyendo distintos territorios del Estado español. Tienen en común el rechazo a las instituciones asilares aunque, como veremos, con diferentes posiciones e importantes controversias.

Veamos concretamente aquellos que, en el marco de los planteamientos anteriormente definidos (1), resultan especialmente importantes para valorar nuestra situación y su deseable evolución.

\section{LA ATENCIÓN COMUNITARIA EN SALUD MENTAL}

Un elemento central para el análisis es el surgimiento y desarrollo de las tendencias que agrupamos bajo la denominación de "atención comunitaria en salud mental" (2-4).

Se trata de un movimiento multiforme surgido en distintos países del entonces denominado "Occidente desarrollado" tras el final de la Segunda Guerra Mundial, partiendo de un conjunto de críticas a la asistencia tradicional basada en hospitales psiquiátricos (5). Críticas estas tanto internas (6-8) como externas (desde la socio- 
logía, la filosofía o la historia (9-12)), pero confluyentes en un cuerpo no siempre homogéneo pero finalmente bastante coherente.

Se trata, en conjunto, de planteamientos que, en referencia a los hospitales psiquiátricos, si bien con distintos matices y énfasis (5):

a) Sitúan su origen y el de la psiquiatría tradicional, de la que fueron soporte organizativo, en un implícito o explícito "mandato" de control social.

b) Analizan su función compleja de protección, pero también de represión y aislamiento.

c) Y ponen de relieve sus efectos iatrogénicos, directamente sobre quienes se ven obligados a "utilizarlos" e indirectamente sobre el estigma social.

Apoyándose en ellos y en distintos movimientos activistas se producen desarrollos más o menos confluyentes $(4,5,13-15)$, desde la psiquiatría de sector francesa (que, sin embargo, mantiene prácticamente intactos sus hospitales) hasta distintos procesos nacionales que combinan políticas de "desinstitucionalización" más o menos coherentes con el desarrollo de estructuras alternativas de atención en "la comunidad”: Estados Unidos, Canadá, Reino Unido, países nórdicos, Italia y, más tardíamente y de manera parcial, España.

En concreto, la superación de las grandes instituciones monográficas se basó en ocasiones en auténticos procesos de desinstitucionalización, pero también y demasiadas veces en cierres administrativos y meras "deshospitalizaciones" (14). Procesos inadecuados que dieron lugar a situaciones de abandono, de falta de hogar ("homelessness") o de "transinstitucionalización" a nuevas o viejas instituciones de servicios sociales o a las prisiones, aunque el origen real de algunos de estos problemas (prevalencia de personas con trastorno mental grave (TMG) en las poblaciones sin hogar o en prisiones) es controvertido (15-18).

El análisis comparativo de distintos procesos (Estados Unidos, Reino Unido, Italia... $(3,4))$ pone de manifiesto algunos requisitos: un marco político, económico y social que impulse o al menos permita la reforma; un compromiso profesional con líderes reconocidos y valores y conocimientos coherentes; y una estructura mínima de servicios sanitarios y sociales públicos en los que incardinar las nuevas estructuras.

La atención comunitaria tiene aspectos organizativos y funcionales diversos, pero también algunas características comunes $(2-4,19)$ que permiten definirla (2) como un verdadero "paradigma tecnológico", articulado en torno a cuatro rasgos básicos:

a) Un enfoque de salud pública con compromiso poblacional y territorial, perspectiva epidemiológica e integración activa en las dinámicas sociales generales.

b) Una filosofía de rehabilitación, enfatizando el funcionamiento social y la autonomía, más allá de los síntomas y las enfermedades, y desarrollando 
una atención integral, desde la promoción de la salud a la inclusión social y la ciudadanía, pasando por el tratamiento y la rehabilitación.

c) La organización de la atención en redes coordinadas de servicios sanitarios y sociales, accesibles, adaptables a las necesidades personales y mayoritariamente situadas en los contextos de vida de la gente.

d) Y el desarrollo de sus actuaciones mediante el trabajo en equipo y la participación de profesionales, personas afectadas, familias y personas cercanas, así como organizaciones sociales más generales.

Es una orientación considerada por la OMS como el estándar a alcanzar (20, 21), que se extiende progresivamente a distintas regiones del mundo, pero que en muchos casos funciona con modelos "mixtos" en los que el peso de los hospitales sigue siendo considerable y que resulta más compatible de lo debido con los modos de intervención del reduccionismo biomédico.

En el aspecto organizativo hay "modelos" distintos, con variantes nacionales y locales $(3,4)$ que van desde el extremo "radical" de los servicios de salud mental de Trieste, con una red descentralizada de centros de salud mental, abiertos 24 horas (22), hasta procedimientos más "pragmáticos", que articulan servicios sanitarios y sociales diversos, como los del Reino Unido o los países nórdicos (23).

Para contrarrestar determinadas insuficiencias de algunos sistemas concretos, desde Estados Unidos primero y extendiéndose progresivamente, surgen procedimientos y equipos específicos para problemas de abordaje más problemático (24), como los equipos de "tratamiento asertivo comunitario" (25), establecidos monográficamente o articulados con las redes ordinarias de servicios, desde el trabajo pionero de Leonard Stein y Mary Ann Test (26) en los años 70 como alternativa al cierre del hospital psiquiátrico de Madison (Estados Unidos). Son los equipos multiprofesionales centrados en la atención personalizada, intensiva, continuada y comunitaria de las personas con mayores dificultades, que, como los de atención a primeros episodios (24) o de apoyo al empleo (27), se intentan incorporar a otros modelos, sin valorar siempre adecuadamente los condicionantes que sus contextos de origen plantean para su integración en el conjunto más amplio de servicios en que se pretenden situar.

\section{La LLAMADA "ANTIPSIQUiaTría”}

Paralelamente al desarrollo de la atención comunitaria, en distintos países y en los años 60 y 70, tuvo lugar la aparición pública de una corriente crítica, tanto con las instituciones asilares como con el propio "corpus teórico" de la psiquiatría, sus estructuras organizativas y sus prácticas profesionales. Enlazando con las críticas al sistema manicomial y bajo el nombre de "antipsiquiatría", es habitual presentarla 
como un movimiento unitario aunque incluye posiciones diversas, alguna de ellas erróneamente adscritas:

a) La original inglesa, representada fundamentalmente por David Cooper y Donald Laing, con algunas experiencias prácticas periféricas a los servicios públicos de salud mental. El primero es responsable de la etiqueta general (“antipsiquiatría”) por su libro de 1967 (28) y el segundo trató de dar consistencia teórica al movimiento $(29,30)$, basándose en la filosofía existencialista de Jean Paul Sartre. Hay en alguna de sus obras críticas brillantes y acertadas de determinadas posiciones de la psiquiatría clásica (29), pero la relevancia de ambos como alternativa coherente flaquea en lo teórico (entre otras cosas, por sus discutibles bases filosóficas y sus sesgos en relación con las patologías de las personas que atendían) y en lo técnico, especialmente tras la aparición de importantes problemas personales.

Ambos fueron considerados protagonistas destacados en la confrontación con la psiquiatría tradicional y sus prácticas institucionales $(31,32)$, y aunque hoy vuelven a reivindicarse desde posiciones profesionales críticas y desde algún sector del movimiento de usuarios y usuarias (33), creo que tienen un valor muy limitado. Rechazan la existencia de enfermedades mentales y el conjunto teórico y técnico de la psiquiatría, mezclando análisis interesantes y afirmaciones cuando menos discutibles; desarrollan, como mucho, experiencias puntuales más o menos privadas y terminan "como el rosario de la aurora", sin haber contribuido de manera significativa a cambiar la psiquiatría tradicional. Eso si no han terminado "dando armas al enemigo"; es decir, a los partidarios de esa psiquiatría "bio-reduccionista" contra la que pretendían luchar.

b) El psicoanalista norteamericano Thomas Szasz $(34,35)$, crítico tanto con la psiquiatría como con los representantes del grupo anterior y sin una clara vinculación, más allá de la práctica privada, con la atención en salud mental, rechaza que las enfermedades mentales sean enfermedades en sentido estricto, comparándolas con una definición estrictamente biológica de enfermedad, y enlaza con las teorías sociológicas de la desviación y el etiquetado (36) sin añadirles, en mi opinión, nada útil ni aclarar qué alternativas tiene para la atención a problemas graves como los que diagnosticamos de esquizofrenia.

c) Y el más global movimiento italiano, articulado en torno a Franco Basaglia y a la asociación profesional Psichiatria Democratica, ocasionalmente adscrito a esta tendencia pero en realidad independiente de la misma, más allá de compartir aspectos de las críticas a la psiquiatría tradicional y a las instituciones asilares (7). 
Comprometidos con la práctica asistencial pública y el desarrollo de prácticas innovadoras ("desinstitucionalización”) y estructuras de servicios comunitarios, promovieron la ley italiana que prohíbe los hospitales psiquiátricos (Ley 180 de 1978) y organizaron servicios comunitarios en diversas regiones del país. De estas experiencias, la más conocida es la de Trieste $(22,37)$, aunque no es ni la única ni la más generalizada y generalizable (38).

A diferencia de los estrictamente "antipsiquiatras", Basaglia no negaba la existencia de la enfermedad ni la importancia de la psicopatología, sino que defendía "ponerla entre paréntesis" (39) hasta poder encontrarse con ella fuera de los muros de las instituciones, sin la carga añadida del institucionalismo.

En conjunto, la llamada "antipsiquiatría” supuso una llamada de atención sobre la injusticia e inadecuación de la psiquiatría tradicional (31), pero, separando la experiencia italiana, es, en mi opinión, una pobre base para construir alternativas de futuro. Creo, por ello, que la vuelta actual hacia sus posiciones muestra más las insuficiencias de algunos modelos críticos que una alternativa real al reduccionismo biomédico.

\section{La "ReHABILITACIÓN PSICOSOCIAL"}

Aunque el término tiene, como veremos, diversos sentidos de uso, hace referencia al desarrollo histórico de una orientación específica de la atención a personas con TMG que, con el nombre de "rehabilitación psicosocial", surgió en los Estados Unidos en los años 70 y se extendió progresivamente en distintos sistemas de atención $(2,32)$, aunque coincidiendo también con estrategias paralelas como, por ejemplo, las desarrolladas en el Reino Unido $(40,41)$. En el contexto estadounidense (cierre generalizado de hospitales psiquiátricos sin generalización paralela de redes alternativas y predominio de una atención psiquiátrica farmacológica), importantes equipos profesionales, vinculados a modelos comunitarios, presentaron como alternativa un conjunto de intervenciones de carácter psicológico y psicosocial orientadas a la "prevención terciaria" (prevención y reducción de las consecuencias funcionales de la enfermedad y apoyo a la integración social de las personas (1)), separada del tratamiento "usual" y conformada habitualmente desde una red de servicios y profesionales específicos. Su "importación” a nuestro país da lugar a algunos debates de interés que hemos tratado de sistematizar en varias ocasiones $(2,42,43)$.

En realidad, es un movimiento complejo y multiforme relacionado a su vez con los de desinstitucionalización y atención comunitaria, y basado en "nuevos" enfoques y conocimientos teóricos sobre las personas con TMG (2), algunos ya mencionados al hablar de epidemiología (1): 
a) Las críticas al concepto de "cronicidad" (44), derivadas de los estudios epidemiológicos de seguimiento de personas con esquizofrenia (1).

b) Los modelos de "vulnerabilidad" y su enfoque biopsicosocial de las necesidades y posibilidades de intervención, centradas en ayudar a personas "vulnerables" a amortiguar los efectos del estrés $(1,2)$.

c) La aplicación de los conceptos de funcionamiento y discapacidad (45, 46), de modo similar a lo relativo a personas con otros problemas de salud de carácter crónico (47).

d) Y la aparición de nuevas intervenciones psicosociales eficaces y efectivas (48).

Entre sus iniciadores en EE. UU hay que mencionar a los equipos liderados por Robert Liberman en Los Angeles (49), William Anthony y colaboradores en Boston (50) y el menos conocido de Mark Spivak, primero allí y posteriormente en Israel (51). Tienen en común, especialmente los dos primeros, el énfasis en la importancia de las habilidades sociales, afectadas por la enfermedad y cuyo desarrollo se convierte en estrategia básica para enfrentarse al estrés y favorecer la interacción social y la vida en la comunidad. Apoyando además esos procesos de resocialización con equipos y dispositivos de soporte en la propia comunidad. Mark Spivak añade una atención especial a los procesos de pérdida y recuperación de competencias sociales, que no dependen solo de la persona enferma. Y todos los modelos han evolucionado hacia un mayor interés por los aspectos de funcionamiento y rehabilitación cognitiva, como hace claramente el programa integrado de los suizos Hans Dieter Brenner y Volker Roder (52).

El desarrollo en Europa de estos modelos y técnicas (por extensión o en paralelo, según los casos) permitió una evolución especialmente interesante. En un contexto muy diferente del americano, con redes razonables de servicios sanitarios y sociales públicos, en el Reino Unido y en los países nórdicos, especialmente en Finlandia, se desarrollan modelos más integrados de servicios donde "la rehabilitación" no funciona como una red separada, contrapuesta o paralela a la "asistencia psiquiátrica”, sino que se integra en redes complejas de servicios. Así, el grupo inglés de John Wing, Douglas Bennett y Geoff Shepherd (40, 41), el de Luc Ciompi en Suiza (53) o los de Yrjö Alanen en Finlandia (54) desarrollan programas integrados de atención a personas con esquizofrenia con un fuerte componente de rehabilitación.

Hay diferencias importantes en la organización de servicios y profesionales, dependiendo de las orientaciones concretas de cada grupo y de su integración en el conjunto de la atención, pero básicamente tienen dos aspectos comunes:

\footnotetext{
${ }^{1}$ Líder de un exitoso proyecto nacional sobre la atención a personas con esquizofrenia, en funcionamiento desde hace más de 30 años y base común de algunas experiencias posteriores (55).
} 
a) Un enfoque general de las necesidades y posibilidades de intervención en apoyo de personas con TMG, planteándose como objetivo ayudarles a vivir en la comunidad contrarrestando sus dificultades, desde modelos de vulnerabilidad $(1,53)$ y desde una consideración global de la discapacidad como producto de la interacción entre síntomas y barreras sociales $(45,46)$.

b) La definición de dos áreas complementarias de intervención: capacitar a las personas para la vida en la comunidad mediante el desarrollo o recuperación del conjunto de habilidades necesarias para ello, y dotarlas de sistemas de soporte en áreas como la vivienda, el empleo, la vida cotidiana y la interacción social (56).

Los debates sobre el tema (43) tienen que ver con las modalidades concretas de integración (relaciones "rehabilitación”/“atención general” y "sanitario"/"social”), pero también con el carácter polisémico del término "rehabilitación” con el que hacemos referencia al menos a 5 aspectos diferentes $(2,43)$, según la entendamos como una filosofía, una estrategia, un nivel de intervención, un tipo de programas o una categoría de servicios y profesionales.

Algunos defendemos desde hace tiempo un enfoque integrador $(2,43)$ para el que la filosofía y la estrategia de rehabilitación deben ser comunes a quienes trabajamos desde modelos de atención comunitaria. Los programas pueden y deben realizarse desde muchos equipos de la red sanitaria y social, y si hay unidades específicas "de rehabilitación", se sitúan en el sector sanitario de la atención.

Pero, además, se ignoran habitualmente las experiencias de desinstitucionalización ("práctica en busca de teoría", en palabras de Benedetto Saraceno (32)) ligadas a los procesos de cierre de hospitales psiquiátricos y de construcción de alternativas a los mismos (en Italia y en otros lugares). Procesos estos que $(5,32)$ :

a) Dentro del hospital y orientándose a su cierre, desarrollaron políticas de humanización y devolución de la dignidad de las personas como paso previo a su "rehabilitación" e inclusión social, por procedimientos más o menos técnicos, pero muy ligados a la vida cotidiana.

b) Fuera del hospital establecen procedimientos concretos de apoyo a la vida en la comunidad, con entrenamientos no demasiado sistemáticos pero muy adaptados a la persona y al contexto, siendo precisamente esos contextos los reales de la vida social y no los del aula o el centro de rehabilitación de muchos procedimientos más "técnicos".

c) Y todo ello junto a una actividad social y política de reivindicación de la ciudadanía y del derecho a la participación social de las personas con TMG, no pretendiendo prioritariamente que "el débil deje de ser débil", sino que todos juntos, "débiles" y "fuertes", podamos coexistir e interactuar en condiciones de igualdad (32). 
Lo que implica, por otro lado, modelos de intervención que hacen efectiva la atención comunitaria, integrando "la rehabilitación" en el conjunto de las intervenciones y no separándola del resto.

\section{EL DESARROLLO DE MOVIMIENTOS ASOCIATIVOS DE LAS PERSONAS IMPLICADAS}

En salud mental, además de las dinámicas propias de las asociaciones profesionales, asistimos a un crecimiento importante de movimientos asociativos de personas implicadas (57), paralelo al de las afectadas por otros problemas de salud. En nuestro campo incluyen dos grupos diferenciados: las familias y las propias personas con problemas de salud mental. Y en nuestro país su desarrollo ha sido más lento y tardío, especialmente en el caso del segundo.

Aunque, al igual que en otros sectores asociativos de personas con problemas de salud y discapacidad, hay una cierta fragmentación en función de distintos diagnósticos o problemas concretos (esquizofrenia, trastorno bipolar, trastornos de personalidad o de la conducta alimentaria...), voy a considerarlos de manera unitaria, aunque centrándome fundamentalmente en los relativos a personas con TMG.

\section{a) El movimiento asociativo de familiares}

Surgió con posiciones ambivalentes con las reformas, con dudas sobre el papel de los nuevos servicios (58-60), pero fue consolidándose posteriormente en posiciones de defensa de los modelos comunitarios, manteniendo un papel de interlocutor crítico con las administraciones y los profesionales, y de lucha contra el estigma ante la población general, generando grandes organizaciones, como la estadounidense National Alliance on Mental Illness (NAMI), la canadiense Family Asociation for Mental Health Everywhere (FAME) o la europea EUFAMI - European Federation of Associations of Families of People with Mental Illness, a la que pertenece la española Salud Mental España (antigua FEAFES). En algunos casos mantiene posiciones críticas con los movimientos de usuarios y usuarias, con la pretensión, implícita o explícita, de asumir también su representación.

Poco a poco han ido definiendo funciones, básicamente en cuatro direcciones más o menos articuladas y con énfasis diferentes según los contextos internos y externos de las organizaciones:

1. El apoyo directo a familiares con funciones de cuidado a personas con TMG.

2. La interlocución crítica con las administraciones y los profesionales.

3. La defensa del colectivo de personas con TMG y de sus familias frente al estigma y la discriminación. 
4. Y el desarrollo de intervenciones más o menos profesionales de atención directa a personas con TMG.

Esta última función, que condiciona frecuentemente una doble dependencia añadida (hacia las administraciones que les pagan y hacia los equipos profesionales que contratan) y resulta discutible como alternativa a la atención pública, es cada vez más frecuente en nuestro país, ante la falta de respuesta pública en muchas comunidades autónomas, pero también por la búsqueda de visibilidad y "poder" de algunos movimientos asociativos.

\section{b) El movimiento de personas con enfermedad mental}

Tiene en la mayoría de los casos un desarrollo posterior y más desigual, al menos en sus fases iniciales, muy dependientes de personas concretas. Adopta nombres diversos, desde el habitual "usuarios y usuarias", pasando por el más crítico de "supervivientes", al de los más radicales, que vuelven a utilizar el tradicional "locos y locas", a semejanza de otros movimientos identitarios de minorías reivindicativas.

Con un desarrollo inicialmente importante en Estados Unidos (61), se extendió progresivamente en Europa (62) y solo más recientemente entre nosotros, pero cada vez con mayor amplitud y consistencia, aunque, en mi opinión, también con algunos sesgos a los que luego aludiré.

Aunque no siempre son bien vistos por los profesionales y las familias, son hoy un elemento imprescindible del campo de la salud mental, por su visión directa, desde la experiencia personal, que les permite desempeñar un papel de apoyo, interlocución y defensa de derechos, específico y diferenciado de los de las familias y los profesionales.

Sus funciones habituales (57) son similares a las referidas para las asociaciones de familiares, pero aquí la primera ("apoyo directo a personas en la misma situación”) y la cuarta ("desarrollo de intervenciones más o menos profesionales de atención") se unifican, con matices propios, en programas y actuaciones de "apoyo mutuo" (63-65) y de desarrollo de estructuras de servicios propios (clubes sociales, clubhouses, centros de día, etc. $(66,67))$. Campo este de interés creciente, con evaluaciones iniciales no muy concluyentes pero en general prometedoras $(65,67,68)$.

Pero para valorar adecuadamente su papel hay que tener en cuenta también algunos sesgos, relativos básicamente a dos temas:

a) La asociación y liderazgo selectivo de personas con mejores perspectivas en términos de recuperación y de capacidad, como en muchos otros movimientos asociativos, pero sesgada probablemente en este caso por un mayor porcentaje de personas con diagnósticos de "trastornos bipolares" que de otras formas de psicosis, como las asociadas habitualmente al diagnóstico de esquizofrenia. 
b) Y la importante diversidad de posiciones que presentan sus grupos en relación al reconocimiento o no de la existencia de enfermedades o trastornos y a su mayor o menor aceptación de los y las profesionales y de los distintos tipos de tratamientos y servicios de atención.

A este respecto, es interesante un estudio realizado hace años en Leipzig por Reinhold Kilian y Matthias Angermeyer (69), en el que identificaban subgrupos de personas con TMG en relación con su disponibilidad para utilizar servicios "de día”: quienes los rechazaban todos, quienes preferían utilizar servicios profesionales (centros de día) y quienes optarían por servicios de movimiento asociativo (clubes sociales). Heterogeneidad esta relacionada con orientaciones de carácter más general y que, como señalaban los autores del estudio, debería tenerse en cuenta al planificar servicios y al proponer itinerarios de atención a personas concretas.

Creo, obviamente, que hay que respetar la existencia y escuchar la palabra de estos movimientos, incluso de los más "radicales", pero sin que ello suponga necesariamente identificarse con todas y cada una de las posiciones de sus diversos componentes, ni dejar de criticar lo que consideremos criticable de las mismas.

\section{Algunos temas “nuevos” asociados al movimiento de usuarios}

Asociados al surgimiento de estos movimientos, encontramos algunos valores importantes que hay que incorporar al marco general de la atención comunitaria, aunque también requieran una lectura crítica. Se trata de los conceptos (objetivos y consignas) de "recuperación” y "empoderamiento" (70), y del énfasis en la protección de los derechos humanos de las personas con este tipo de problemas, en la sociedad en general y en su relación con servicios y profesionales (71).

Son temas que ni son nuevos ni dependen únicamente de la presencia de las asociaciones, pues su origen (no siempre con estas denominaciones) se relaciona, de manera más general, con los movimientos de desinstitucionalización, rehabilitación y atención comunitaria. Pero las formulaciones de las personas directamente afectadas añaden matices, dimensiones y aspectos específicos a tener en cuenta.

\section{a) La "recuperación"}

Más allá de definiciones profesionales (72), centradas en la mejoría sintomatológica y funcional que, en grado variable, alcanza al menos un número importante de personas con TMG (según los datos epidemiológicos ya referidos (1) y ampliamente compilados por Richard Warner (73)), la visión del tema desde los movimientos de usuarios y usuarias enfatiza una dimensión personal tanto del proceso de enfermar como de su posible superación. Esta se entiende precisamente como la recuperación o 
consecución de una vida razonablemente satisfactoria, incluso aunque siga habiendo manifestaciones sintomatológicas y funcionales, a la vez que se recalca el papel de las dinámicas no profesionales, como el esfuerzo personal y los apoyos del entorno (72).

Se ha señalado, sin embargo, el riesgo nada lejano de instrumentación del tema desde posiciones "neoliberales" (74), desde las que parecen decirnos que no hacen falta servicios, sino sobre todo "esfuerzo personal". Y también la variada y discutible aplicación del concepto a los diversos tipos de problemas (75), más difícil (y por tanto suponemos que con menor porcentaje de quienes lo consiguen, aunque aquí faltan datos cuantitativos) en el caso, por ejemplo, de personas con esquizofrenia que en el de personas con trastornos bipolares.

\section{b) El "empoderamiento"}

Concepto importante (70) que enfatiza el papel activo, individual y colectivo, de las personas afectadas, con implicaciones en la relación profesional/usuario y usuaria, planteando la necesidad de una mayor simetría y enlazando con temas como las decisiones compartidas y las voluntades anticipadas $(76,77)$. En continuidad y no en ruptura con la reivindicación de ciudadanía de la atención comunitaria, su aplicabilidad es también variable, según los distintos tipos de problemas y situaciones concretas de las personas con TMG.

\section{c) La problemática relacionada con los derechos humanos}

Aspecto clave frente a políticas socialmente restrictivas (rechazo y discriminación general, legislación restrictiva civil y penal, incluyendo las restricciones de la capacidad legal, además de diversas formas de discriminación administrativa) y de la propia atención "tradicional", frecuentemente caracterizada por una importante falta de respeto humano en la relación y por el uso abusivo de distintas intervenciones restrictivas de la libertad individual (sujeción mecánica, internamientos involuntarios y tratamientos ambulatorios involuntarios) $(71,78)$.

Pero, más allá de la referencia ritual a la Convención de las Naciones Unidas sobre los derechos de las personas con discapacidad, requiere también un análisis crítico y pormenorizado que no olvide algunas realidades complejas $(79,80)$ :

a. Por un lado, que en nuestro campo hay a veces conflictos de derechos (libertad, protección de la salud, seguridad propia y ajena) que obligan a contemplar algunas medidas restrictivas de la libertad individual, lo que implica el control de la Administración de Justicia. Es el caso de los internamientos involuntarios, aunque no de su injustificada extensión al tratamiento ambulatorio (78). 
b. Y, por otro, que la integración en la sociedad tiene contradicciones, como muestra el complejo tema de las prisiones en relación con la comisión de delitos por parte de personas con TMG $(18,81)$. La prisión no es un entorno especialmente recomendable para personas con TMG (en general para nadie, claro), pero tampoco debe excluirse su ingreso en la misma, si hablamos de normalización, cuando así corresponde según las leyes. Lo que no implica que no deban adecuarse las condiciones del sistema penitenciario ni dejar de explorar posibilidades alternativas de atención en instituciones sanitarias, siempre que queden claras las competencias respectivas y no se pretenda convertir los servicios sanitarios en una extensión del aparato penitenciario, como sucedía (y sucede) con los hospitales psiquiátricos.

\section{La SITUACión EN EsPaña}

Las descripciones y análisis resumidos anteriormente hacen referencia a la situación general del campo de la salud mental y son en gran parte aplicables a nuestra situación concreta, más allá de algunas referencias territoriales específicas. Pero, dentro del panorama general, la situación española tiene aspectos y matices propios que hay que tener en cuenta.

Desde la caótica situación heredada de la Dictadura y de la formulación oficial de la Reforma, se generó una gran diversidad de situaciones en los diversos territorios, en pocos de los cuales se consiguió una transformación importante, al menos, de las estructuras tradicionales de atención, incluyendo la superación de los hospitales psiquiátricos. Es el caso fundamentalmente de Andalucía, Asturias, quizás Navarra y al menos una parte de Madrid (82-85).

En conjunto, el proceso ha sido bastante deficiente, considerando aspectos como:

a) La política seguida con los hospitales psiquiátricos, que en su mayoría siguen existiendo con un número de camas por encima de las 12.000 (más del $40 \%$ de las existentes en $1985^{2}$ ) y, en los casos en que se cerraron, mediante procesos de cierre que han sido también muy desiguales (5).

b) El desarrollo de servicios alternativos, notablemente desigual, insuficiente y sesgado en los sanitarios por basarse mayoritariamente en equipos am-

\footnotetext{
${ }^{2}$ Cifras imprecisas debido a procedimientos de "maquillaje", con cambios de nombre, catalogación y/o adscripción administrativa de algunos centros, que salen así artificialmente del registro sanitario (Catalogo Nacional de Hospitales y Estadística de Centros de Atención Especializada). Los datos, actualizados a 2017, proceden de mi presentación ("Los hospitales psiquiátricos que no se quieren mirar") a la mesa de la Sección de Rehabilitación de la AEN del XIII Congreso Mundial de la WAPR (Madrid, 6 de julio de 2018).
} 
bulatorios de salud mental y unidades "de agudos" en hospitales generales, coexistiendo con los restos del sistema manicomial.

c) El desarrollo más escaso y poco homogéneo aún en los no sanitarios (residenciales, de empleo...), mayoritariamente en manos de organizaciones privadas lucrativas o formalmente "no lucrativas". Hay que mencionar las excepciones de Andalucía y Madrid en la cobertura de recursos residenciales, de empleo y de soporte comunitario, aunque solo en el caso de Andalucía los servicios son públicos también en su gestión $(43,56)$.

d) El modelo de actuación, más orientado por las posiciones del reduccionismo biomédico que por las de la atención comunitaria, aunque no tenemos datos cuantitativos que nos permitan medir esa preponderancia, más allá de la experiencia cotidiana en los servicios y de la capacidad de atracción de las asociaciones profesionales del sector.

e) La persistencia de legislaciones restrictivas, en temas civiles (incapacitaciones y medidas de protección) y penales (medidas de seguridad), con intentos periódicos de aumentar las medidas coercitivas.

f) Con movimientos asociativos en crecimiento, más extenso el de familiares y en proceso de extensión el de usuarios y usuarias, aunque con posiciones muy diferentes en su seno, algunas, en mi opinión, poco favorables para conseguir alianzas críticas capaces de establecer "bloques históricos" favorables al cambio.

g) Y sin una política general clara, ni a nivel estatal ni en el de la inmensa mayoría de sus comunidades autónomas. Ausencia esta que llega al extremo de carecer de un mínimo sistema de información común que permita hacerse una idea cuantitativa de la situación en cada una de ellas ni en el conjunto del Estado.

\section{Algunas Conclusiones mirando al futuro}

El objetivo general del apretado recorrido realizado era mostrar que es necesario y posible proponer una alternativa razonable al reduccionismo biomédico, sin tener que embarcarse en las posiciones de algunos movimientos y personalidades formalmente "críticos" del mismo. Si las pobres e inadecuadas respuestas del reduccionismo a las necesidades de las personas con problemas de salud mental deben generar el rechazo y la búsqueda de alternativas teóricas y técnicas, ello no implica que tengamos que aceptar acríticamente las que nos proponen desde la "postpsiquiatría" en alianza con algunos representantes de movimientos extremos de personas directamente afectadas por los problemas. Por el contrario, más que recurrir a posiciones herederas de la antipsiquiatría residual y, más en general, de las confusiones del 
postmodernismo, creo que cabe una respuesta basada en lo que hemos ido trabajosamente aprendiendo, en la teoría y en la práctica, en estas décadas de importantes transformaciones, pese a sus errores e insuficiencias.

Así, desde posiciones filosóficas materialistas en ontología y realistas en epistemología (1), hemos tratado de valorar críticamente tanto las aportaciones como los sesgos que ha supuesto la adscripción de nuestro campo al de los sistemas sanitarios, así como algunos movimientos teóricos y técnicos sucedidos en las últimas décadas, especialmente la atención comunitaria y la rehabilitación psicosocial. Y, como resultado de todo ello, cabe situar el tema a partir de las siguientes afirmaciones:

a) Que, en nuestras sociedades, un número significativo de personas presenta determinadas conductas (en sentido amplio, es decir, incluyendo comportamientos, cogniciones y sentimientos) que podemos considerar estadísticamente "no normales" y funcionalmente "anómalas", por cuanto suponen sufrimiento personal y del entorno, generan dificultades en la vida personal y social, y responden a modos de funcionamiento mental diferenciados de los habituales. $\mathrm{Y}$ eso no es una invención de las disciplinas y profesionales de la salud mental o de determinados sectores sociales poderosos, sino una realidad independiente de los mismos, aunque condicionada en modo diverso por ellos.

b) Que, para dar cuenta de dichas conductas y tratar de ayudar a quienes las desarrollan (las personas "sujetos" de las mismas), las disciplinas teóricas y técnicas de la salud mental siguen y deben seguir beneficiándose de su inclusión mayoritaria en el más amplio campo sanitario, aunque esa adscripción tenga carácter histórico, socialmente condicionado e introduzca importantes sesgos y riesgos, de los que son ejemplo las formas teóricas y técnicas del reduccionismo biomédico. Modelo este desgraciadamente habitual de la psiquiatría, que no puede identificarse como el "científico", "tecnológico" o "basado en la evidencia", como pretenden hacernos creer sus portadores y les conceden, generosa e injustificadamente, algunos de sus críticos profesionales y no profesionales ${ }^{3}$.

c) Que, en ese sentido, son especialmente importantes las perspectivas de la epidemiología y la salud pública, enfocando los aspectos sociales de los procesos de salud y enfermedad y la organización global de los sistemas de atención dentro de las dinámicas específicas de las sociedades humanas.

d) Que, en el terreno más individual o personal, también la perspectiva sanitaria es útil a la hora de subrayar la importancia de construir una real psico(pato)logía, más allá de las descripciones "botánicas" de síntomas que

${ }^{3}$ Como coartada, en muchas ocasiones, para proponer alternativas fuera del campo de las ciencias, las tecnologías y la información evaluativa de nuestras intervenciones. 
nos deja la psiquiatría "clásica" o las recetas para supuestos diagnósticos de los sistemas internacionales habituales.

e) Que, en lo que respecta a la organización, sigue siendo válida la perspectiva de la atención comunitaria en salud mental, apoyada en la atención primaria y con un fuerte componente de rehabilitación. En relación con las personas con TMG, es básica su apuesta por redes de servicios sanitarios, sociales, laborales y educativos, cuya actuación se basa en la cooperación entre equipos multiprofesionales y organizaciones sociales, especialmente las que agrupan por un lado a familiares y por otro a usuarios y usuarias.

f) Que, en relación con la atención directa a las personas, especialmente a aquellas con TMG, es necesario, en bastantes ocasiones, ir más allá del mero acompańamiento en las crisis, consideradas erróneamente por algunos y algunas como experiencias automáticamente positivas. Ese "ir más alla”, mediante intervenciones profesionales, debe basarse en el establecimiento de una alianza terapéutica entre el equipo y la persona afectada, con implicación siempre que sea posible de figuras relevantes del entorno (familiares, amigos y amigas, pero también de otras personas cercanas o con experiencias similares). Desde ahí deberían fijarse objetivos basados en valores de recuperación, inclusión social y ciudadanía, y utilizando para alcanzarlos procedimientos técnicos con el mayor grado de "evidencia" disponible.

g) Que, en ese proceso de ayuda, los profesionales deben combinar en cada caso los conocimientos generales de la psico(pato)logía (características generales del tipo de trastorno que la persona presenta y que se resume en un diagnóstico genérico) con los específicos de dicha persona y de su entorno (que permiten concretar dicho diagnóstico), estableciendo también aquí un "diálogo abierto" en el que las distintas perspectivas (persona, profesional, entorno) deben combinarse pero no confundirse.

h) Que, en concreto, el hecho de que la persona que presenta el problema tenga su propia perspectiva y visión del mismo y de sus deseables soluciones, y el de que esta perspectiva deba tenerse en cuenta no significa que sea la única a considerar, ni siempre la que deba tener la última palabra. Las relaciones profesional/usuario o usuaria no son nunca perfectamente simétricas, la persona que presenta el trastorno no es la única afectada y no podemos olvidar que, en no pocas ocasiones, el trastorno afecta a su capacidad para captar la realidad y analizar coherentemente sus alternativas. Tratar de consensuar estas no exime al o a la profesional de sus responsabilidades en cuanto tal (80).

i) Que, también en relación con la intervención profesional, hay que tener en cuenta que el objetivo es apoyar la recuperación de la persona, para lo 
que hay que analizar críticamente las posibilidades de la situación, escuchar y valorar las perspectivas de la persona y el entorno, entender las dinámicas generadas y ayudar a que se orienten en la dirección más razonable. Apoyando humana y técnicamente el proceso siempre complejo de hacer operativa esa dirección.

j) Y que, para ello, son utilizables distintos procedimientos técnicos, desde un uso razonable de algún fármaco y de técnicas psicoterapéuticas solventes (dirigidas a la persona y a su entorno inmediato) a los programas de rehabilitación y apoyo social en la comunidad. Incluyendo el apoyo de otras personas con experiencia directa en los problemas y en los procesos de recuperación.

Situándonos en el caso concreto de nuestro país, un "futuro deseable" significaría, para mí, moverse en dirección a:

1. Acabar realmente la Reforma Psiquiátrica, lo que significa, por un lado, superar definitivamente las grandes instituciones monográficas, incluyendo los viejos hospitales psiquiátricos con ese u otro nombre y las nuevas instituciones no menos totales establecidas en algunos lugares en estos años. Y, por otro, hacer más homogénea y mejor dotada una organización alternativa.

2. En relación precisamente con la organización de la atención, potenciar una estructura y funcionamiento intersectorial "en red":

a) Manteniendo en el sistema sanitario los dispositivos y equipos profesionales de ese carácter, y tratando en concreto de:

a. Potenciar los equipos generales, polivalentes, cercanos a la población y con modelos de atención asertiva, continuada y participativa.

b. Adecuar las unidades de hospitalización de corta estancia dentro del contexto hospitalario, reduciendo al máximo su uso mediante el trabajo de los equipos comunitarios y de alternativas domiciliarias y residenciales.

c. Complementar la red con unidades más especializadas, como pueden ser hospitales de día, unidades de rehabilitación o estructuras activas de media y larga estancia (del tipo de las comunidades terapéuticas andaluzas (86)), así como equipos más especializados en el abordaje de problemas o situaciones concretas.

d. Pero estableciendo siempre un funcionamiento "en red", con un papel central de los equipos territoriales generalistas en conexión con la Atención Primaria y superando los modelos de "derivación".

b) Complementar la atención sanitaria con estructuras de soporte para el alojamiento, el empleo y el apoyo no sanitario en la vida cotidiana, articuladas con ella. 
c) Dar una mejor respuesta al tema de las prisiones y una alternativa a los hospitales psiquiátricos penitenciarios, en línea con los desarrollos italianos recientes (87).

d) Apoyar la participación de las asociaciones de usuarios y usuarias en la atención, dentro y fuera de las estructuras profesionales.

e) Y establecer espacios territorializados de coordinación entre profesionales, usuarios y familiares.

3. Y, en relación con los y las profesionales, será necesario desarrollar el modelo de atención comunitaria en sus implicaciones clínicas, lo que implica:

a) Mantenerse al día de la evolución de los conocimientos generales desde la biología, la sociología y la psicología para continuar trabajando en la elaboración de una psico(pato)logía que merezca ese nombre.

b) Definir una clínica basada en la relación, integradora de los conocimientos teóricos y técnicos (psicopatología, epidemiología, relatos de las personas afectadas...) y respetuosa con los derechos e iniciativas de las personas.

c) Apoyar la investigación sobre evaluación de nuestras intervenciones, acordes con los objetivos de recuperación.

d) Desarrollar programas de formación igualmente acordes con esos planteamientos.

e) Y potenciar una organización profesional independiente (de la industria y de los otros movimientos asociativos) como interlocutor diferenciado en los distintos "diálogos" que necesitamos mantener.

Es verdad que, como comentaba al principio del artículo anterior (1) citando a Josep Fontana, "el futuro es un país extraño" (88) y las perspectivas de avance no son en general especialmente optimistas, pero cabe aquí también recordar a Antonio Gramsci y llamar con él a moderar "el pesimismo de la inteligencia con el optimismo de la voluntad" (89).

\section{BibLIOGRAFÍA}

(1) López M. Mirando atrás para seguir avanzando. Una reflexión crítica sobre el pasado y el presente de la atención en salud mental (I). Rev Asoc Esp Neuropsiq. 2019; 39(136): 89-116.

(2) López M, Laviana M. Rehabilitación, apoyo social y atención comunitaria a personas con trastorno mental grave. Algunas propuestas desde Andalucía. Rev Asoc Esp Neuropsiq. 2007: 99:187-223.

(3) Thornicroft G, Deb T, Henderson C. Community mental health care worldwide: current status and further developments. World Psychiatry 2016; 15 (3): 276-286. 
(4) Rosen A, O'Halloran P, Mezzina R. International trends in community mental health services. En: McQuistion HL, Sowers WE, Ranz JM, Feldman JM (Eds.). Handbook of Community Psychiatry. New York: Springer, 2012; pp. 389404.

(5) López M, Laviana M, García-Cubillana P. Los hospitales psiquiátricos en la(s) reforma(s). Notas para orientar una investigación necesaria. En: Pérez F (Coord.). Dos décadas tras la Reforma Psiquiátrica. Madrid: AEN, 2006; pp. 239-276.

(6) Barton R. La neurosis institucional. Madrid: Paz Montalvo, 1974.

(7) Basaglia F. La institución negada. Informe de un hospital psiquiátrico. Barcelona: Barral, 1972.

(8) Wing JK, Brown GB. Institutionalism and schizophrenia. London: Cambridge University Press, 1970.

(9) Castel R. L'ordre psychiatrique. L'âge d'or de l'aliénisme. Paris: Editions de Minuit, 1976.

(10) Foucault M. Histoire de la folie à l'âge classique. Paris: Gallimard, 1972.

(11) Goffman E. Internados. Ensayos sobre la situación social de los enfermos mentales. Buenos Aires: Amorrortu, 1970.

(12) Scull AT. Decarceration: community treatment and the deviant -A radical view. Englewood Cliffs, NJ: Prentice-Hall, 1977.

(13) Desviat M. Cohabitar la diferencia. De la Reforma Psiquiátrica a la salud mental colectiva. Madrid: Grupo 5, 2016.

(14) Fakhoury W, Priebe S. The process of deinstitutionalization: an international overview. Curr Opin Psychiatry. 2002; 15:187-192

(15) Bagenstos SR. The past and future of deinstitutionalization litigation. Law \& Economics Working Papers. 2012, 46. https://repository.law.umich.edu/law_econ_current/art46.

(16) Fazel S, Khosla V, Doll H, Geddes J. The prevalence of mental disorders among the homeless in western countries: systematic review and meta-regression analysis. PLoS Medicine. 2008; 5(12): e225.

(17) Main T. How to think about homelessness: balancing structural and individual causes. J Soc Distress Homeless. 1998; 7(1): 41-54

(18) López M, Saavedra FJ, López A, Laviana M. Prevalencia de problemas de salud mental en varones que cumplen condena en centros penitenciarios de Andalucía (España). Rev Esp Sanid Penit. 2016; 18: 76-85

(19) Desviat M, Moreno A (eds.). Acciones de salud mental en la comunidad. Madrid: AEN, 2012.

(20) Organización Mundial de la Salud. Informe sobre la salud mental en el mundo 2001. Salud mental: nuevos conocimientos, nuevas esperanzas. Ginebra: OMS, 2001.

(21) López M. Políticas de salud mental en la Organización Mundial de la Salud. En: Ansean A (ed.). Manual de gestión clínica y sanitaria en salud mental. Madrid: Edicomplet, 2012; pp. 37-51.

(22) Mezzina R. Community mental health care in Trieste and beyond: an "open doorno restraint” system of care for recovery and citizenship. J Nerv Ment Dis. 2014; 202 (6):440-445. 
(23) Knapp M, McDaid D, Mossialos E, Thornicroft G (ed.). Salud mental en Europa: políticas y práctica. Líneas futuras en salud mental. Madrid: Ministerio de Sanidad y Consumo, 2007.

(24) Burns T. Community mental health teams. A guide to current practices. Oxford: Oxford University Press, 2004.

(25) Rosen A, Mueser KT, Teesson M. Assertive community treatment - issues from scientific and clinical literature with implications for practice. J Rehabil Res Dev. 2007; 44(6):813-25.

(26) Stein LI, Test MA. Alternative to mental hospital treatment. I: conceptual model, treatment program and clinical evaluation. Arch Gen Psychiatry. 1980; 37:392397.

(27) López M, Laviana M, González S. Rehabilitación laboral y programas de empleo. En: Pastor A, Blanco A, Navarro D (coords.). Manual de rehabilitación del trastorno mental grave. Madrid: Síntesis, 2010.

(28) Cooper D. Psiquiatría y antipsiquiatría. Buenos Aires: Paidós, 1971.

(29) Laing RD. El yo dividido. México DF: Fondo de Cultura Económica, 1964.

(30) Laing RD, Esterson A. Cordura, locura y familia. México DF: Fondo de Cultura Económica, 1967.

(31) Desviat M. La antipsiquiatría: crítica de la razón psiquiátrica. Norte de Salud Mental 2006; 25: 8-14.

(32) Saraceno B. El fin del entretenimiento. Manual de rehabilitación psiquiátrica. Madrid: AEN, 2014.

(33) Burstow B. Antipsychiatry revisited: toward greater clarity. Disponible en: madinamerica.com/2014/10/antipsychiatry-revisited-toward-greater-clarity/

(34) Szasz T. Schizophrenia: the sacred symbol of psychiatry. New York: Syracuse University Press, 1988.

(35) Szasz T. El mito de la enfermedad mental. Buenos Aires: Amorrortu, 1976.

(36) Becker H. Outsiders. Hacia una sociología de la desviación. Buenos Aires: Siglo XXI Editores, 2009.

(37) Rotteli F. L'Institutione inventata/ Almanaco Trieste 1971-2010. Merano: Edizioni alpha beta Verlag, 2015.

(38) Fioritti A. Is freedom (still) therapy? The 40th anniversary of the Italian mental health care reform. Epidemiol Psychiatr Sci. 2018,16:1-5.

(39) Basaglia F. Un problema di psichiatria instituzionale. L'esclusione come categoria socio-psichiatrica. En: Basaglia F. Scritti I. 1959-1968. Torino: Einaudi, 1981; pp. 12-28.

(40) Watts FN, Bennett DH (ed.). Theory and practice of psychiatric rehabilitation (2a edición). Chichester: Wiley and Sons, 1991.

(41) Wing JK, Morris B (ed). Handbook of psychiatric rehabilitation practice. Oxford: Oxford University Press, 1981.

(42) López M. Diez referencias destacadas en rehabilitación psicosocial. Anuario de Psicología Clínica y de la Salud, 2011; 7:9-14.

(43) López M, Laviana M. Rehabilitación psicosocial y atención comunitaria: algunas consideraciones críticas y una propuesta de guion para el debate. Rev Asoc Esp Neuropsiq. 2017; 37(131): 257-276. 
(44) Harding CM, Zubin J, Strauss JS. Chronicity in schizophrenia: revisited. Br J Psychiatry Supplement, 1992;18:27-37.

(45) Mulvany J. Disability, impairment or illness? The relevance of the social model of disability to the study of mental disorder. Sociol Health Illn. 2000; 22 (5): 582601.

(46) Vázquez-Barquero JL, Herrera S, Vázquez ME, Uriarte M, Herrán A, Grupo Cantabria en Discapacidades. La discapacidad: modelos interpretativos y su influencia en el nuevo sistema de clasificación de discapacidades de la Organización Mundial de la Salud (CIDDM-2). Arch Psiquiatr. 2000;63(1):5-20.

(47) Goering S. Rethinking disability: the social model of disability and chronic disease. Curr Rev Musculoskelet Med. 2015, 8:134-138.

(48) Mueser KT, Deavers F, Penn DL, Cassisi JE. Psychosocial treatments for schizophrenia. Annu Rev Clin Psychol. 2013; 9: 465-497.

(49) Liberman R. Recovery from disability: manual of psychiatric rehabilitation. Arlington: American Psychiatric Publishing, 2008.

(50) Anthony WA, Cohen M, Farkas M, Gagne C. Psychiatric rehabilitation (2a edición). Boston: Center for Psychiatric Rehabilitation. 2002.

(51) Spivak M. Introduzione alla rehabilitazione sociale: teoria, tecnología e metodi di intervento. Rivista Sperimentali di Freniatría. 1987;11:522 -574.

(52) Roder V, Brenner HD, Hodel B, Kienzle N. Terapia integrada de la esquizofrenia. Barcelona: Ariel, 1996.

(53) Ciompi L. Afecto-lógica. El vínculo entre el afecto y la lógica. Una contribución al estudio de la esquizofrenia. Madrid: Fundación para la Investigación y el Tratamiento de la Esquizofrenia y otras Psicosis, 2010.

(54) Alanen YO. La esquizofrenia. Sus orígenes y su tratamiento adaptado a las necesidades del paciente. Madrid: Fundación para la Investigación y el Tratamiento de la Esquizofrenia y otras Psicosis, 2003.

(55) Aaltonen J, Seikkula J, Lehtinen K. The comprehensive open-dialogue approach in Western Lapland: I. The incidence of non-affective psychosis and prodromal states. Psychosis, 2011; 3: 179-191

(56) FAISEM. Soportes para la ciudadanía. Los programas de apoyo social a personas con trastornos mentales graves en Andalucía. Sevilla: FAISEM, 2012.

(57) Rogers A, Pilgrim D. A sociology of mental health and illness (5a edición). Berkshire: McGraw Hill, 2014.

(58) UCLA. Family advocacy: a nuclear movement. UCLA History of Public mental Health. Disponible en: http://histpubmh.semel.ucla.edu/stories.

(59) Department of Health and Human Services. Mental health: A report of the Surgeon General. Rockville: National Institute of Mental Health, 1999.

(60) Dougoud L. Asociaciones de familiares de enfermos mentales. En: García J, Espino A, Lara L (ed.). La psiquiatría en la España de fin de siglo. Madrid: Díaz de Santos, 1978; pp. 279-284

(61) McLean A. The mental health consumers/survivors' movement in the United States. En: Scheid TL, Brown TN (ed.). A handbook for the study of mental health. Social contexts, theories and systems (2a edition). Cambridge: Cambridge University Press, 2010; pp. 461-477. 
(62) Rose D, Lucas R. Movimiento de las personas usuarias y supervivientes en Europa. En: Knapp M, McMaid D, Mossialos E, Thornicroft G. Salud mental en Europa: políticas y práctica. Líneas futuras en salud mental. Madrid: Ministerio de Sanidad y Consumo y Observatorio Europeo de Políticas y Sistemas Sociales, 2007.

(63) Mead S, Hilton D, Curtis L. Peer support: a theoretical perspective. Psychiatr Rehabil J. 2001; 25 (2): 125-141.

(64) En Primera Persona. Recuperación y ayuda mutua. Guía de apoyo para el moderador de grupos de ayuda mutua. Federación Andaluza de Asociaciones de Usuarios/Usuarias de Salud Mental "En primera persona", 2014. (www.enprimerapersonaandalucia.org)

(65) Lloyd-Evans B, Mayo-Wilson E, Harrison B, Istead H, Brown E, Pilling S, et al. A systematic review and meta-analysis of randomised controlled trials of peer support for people with severe mental illness. BMC Psychiatry. 2014; 14:39.

(66) Tanenbaum SJ. Mental health consumer-operated services organizations in the US: citizenship as a core function and strategy for growth. Health Care Anal. 2011;19(2):192-205.

(67) Substance Abuse and Mental Health Services Administration. Consumer-Operates Services: the evidence. HHS Pub. No SMA-11-4633. Rockville MD: CMHS-SAMHSA, 2011.

(68) Pitt V, Lowe D, Hill S, Prictor M, Hetrick SE, Ryan R, Berends L. Consumer-providers of care for adult clients of statutory mental health services. Cochrane Database System Rev. 2013; 28(3):CD004807

(69) Kilian R, Lindenbach I, Löbig U, Uhle M, Angermeyer M. Self-perceived social integration and the use of day centers of persons with severe and persistent schizophrenia living in the community: a qualitative analysis. Soc Psychiatry Psychiatr Epidemiol. 2001; 36(11):545-552

(70) Laviana M. Empoderamiento y recuperación en salud mental. En: Ansean A (ed.). Manual de gestión clínica y sanitaria en salud mental. Madrid: Edicomplet, 2012; pp. 213-222.

(71) Organización Mundial de la Salud. Manual de recursos de la OMS sobre salud mental, derechos humanos y legislación. Ginebra: Organización Mundial de la Salud, 2006.

(72) Bellack AS. Scientific and consumer models of recovery in schizophrenia: concordance, contrasts, and implications. Schizophr Bull. 2006; 32(3):432-442.

(73) Warner R. Recovery in schizophrenia. Psychiatry and political economy (3a edición). Hove: Brunner-Routledge, 2004.

(74) Braslow JT. The manufacture of recovery. Annu Rev Clin Psychol. 2013; 9:781-809.

(75) Uriarte JJ, Vallespí A. Reflexiones en torno al modelo de recuperación. Rev Asoc Esp Neuropsiq. 2017; 37(131): 241-256.

(76) Cosh S, Zenter N, Ay ES, Loos S, Slade M, de Rosa C, et al. Clinical decision making and mental health service use among persons with severe mental illness across Europe. Psychiatr Serv. 2017;68(9): 970-974.

(77) Escuela Andaluza de Salud Pública. Planificación anticipada de decisiones en salud mental. Guía de apoyo para profesionales y personas usuarias de los servicios de salud mental. Granada: EASP, 2015. 
(78) López M, Laviana M. Intervenciones no voluntarias en salud mental. Consideraciones sobre la propuesta de autorización judicial del tratamiento ambulatorio involuntario. Rehabilitación Psicosocial. 2007; 4:28-36.

(79) Szmukler G, Daw R, Callard F. Mental health law and the UN Convention on the Rights of Persons with Disabilities. Int J Law Psychiatry. 2014; 37: 245-252.

(80) Freeman MC, Kolappa K, Caldas de Almeida JM, Kleinman A, Makhashvili N, Saraceno B, et al. Reversing hard won victories in the name of human rights: a critique of the general comment on article 12 of the UN Convention on the Rights of Persons with Disabilities. Lancet psychiatry. 2015; 2: 844-850.

(81) López M, Laviana M, López A. Estigma social, violencia y personas con trastornos mentales graves. En: Márkez I, Fernández A, Pérez P (Coord.). Violencia y salud mental. Salud mental y violencia institucional, estructural, social y colectiva. Madrid: AEN, 2009.

(82) González de Chávez M (coord.). La transformación de la asistencia psiquiátrica. Madrid: Mayoría, 1980.

(83) García J, Espino A, Lara L (ed.). La psiquiatría en la España de fin de siglo. Madrid: Díaz de Santos, 1978.

(84) Pérez F (coord.). Dos décadas tras la Reforma Psiquiátrica. Madrid: AEN, 2006.

(85) Desviat M. La Reforma Psiquiátrica. Madrid: Dorsa, 1994.

(86) Laviana M. Una comunidad terapéutica de salud mental como espacio para la recuperación. El papel de una unidad de media y larga estancia en una red comunitaria pública. Documentación para el Taller no 1 del XXI Curso Anual de Esquizofrenia. Madrid: Fundación para la Investigación y Tratamiento de la Esquizofrenia y otras Psicosis, 2016.

(87) Facchi E, Cardamome G. La salute mentale publicca riformata dalla legge 81: criticità e prospettive. Gruppi, 2017;1: 41-49.

(88) Fontana J. El futuro es un país extraño. Una reflexión sobre la crisis social de comienzos del siglo XXI. Barcelona: Ediciones de Pasado y Presente, 2013.

(89) Gramsci A. Discorso agli anarchici. L’Ordine Nuovo 1920; 1(43): 3-10. 